\section{Doubts about Targretin in AD}

\section{By Lev Osherovich, Senior Writer}

Four independent academic teams have called into question ${ }^{1-4}$ some conclusions from a 2012 paper by Case Western Reserve University School of Medicine researchers that suggested the cancer drug Targretin bexarotene could be useful for treating Alzheimer's disease. ${ }^{5}$ The new studies failed to replicate some aspects of the original work, but the main conclusions-that Targretin reduces levels of soluble $\beta$-amyloid and improves cognitive function in mice-appear to stand.

A clearer picture should emerge in early 2014, when researchers from the Cleveland Clinic Lou Ruvo Center for Brain Health are expected to report findings from a Phase II trial of bexarotene to treat $\mathrm{AD}$.

Targretin is marketed by Eisai Co. Ltd. to treat cutaneous T cell lymphoma (CTCL) and went off patent last year. The compound is an agonist of retinoid X receptor (RXR), a transcription factor that controls expression of proteins involved in lipid transport in the brain. Defects in lipid transport contribute to $\mathrm{AD}$.

In the original study, a team led by Case Western professor of neurosciences and neurology Gary Landreth examined the effect of agonizing RXR on $\beta$-amyloid $(\mathrm{A} \beta)$ clearance and cognition in a mouse model for AD.

In one commonly used mouse model for $\mathrm{AD}$, Targretin led to higher levels of apolipoprotein $\mathrm{E}$ (ApoE), a lipid-ferrying protein that counteracts $A \beta$ accumulation, than did vehicle. Targretin-treated mice also had lower levels of soluble $A \beta$ and $A \beta$ plaques in the brain and performed better in tests of cognition and memory than vehicle-treated controls.

Because of the striking effect of Targretin on A $\beta$ plaques-about a $75 \%$ decrease compared with controls-independent academic teams at the University of Pittsburgh, the University of Florida College of Medicine, the Catholic University Leuven and a consortium of researchers at The University of Chicago, Harvard Medical School, the University of Tuebingen, the Northwestern University Feinberg School of Medicine and the Washington University in St. Louis School of Medicine tried to replicate Landreth's study.

In each case, the teams examined the effects of Targretin or generic formulations of bexarotene in a wide range of mouse models for $\mathrm{AD}$ as well as in dogs.

None of the four teams saw a statistically significant decrease in $A \beta$ plaque levels after treatment. Two of the four teams found that bexarotene decreased levels of soluble $A \beta$ in cerebrospinal fluid or interstitial fluid compared with vehicle, whereas the other two teams saw no statistically significant change in soluble $\mathrm{A} \beta$.
The Leuven team, headed by Bart De Strooper, professor of human genetics, tested the effects of bexarotene on cognition and memory and saw that treated animals had modestly better performance in an assay of social memory compared with animals given vehicle.

De Strooper noted in his technical comment in Science that the drug's toxicity led to behavioral abnormalities that made cognitive assays difficult to interpret.

In contrast, a team co-led by Radosveta Koldamova and Iliya Lefterov, both associate professors of environmental and occupational health at the University of Pittsburgh, showed that bexarotene improved memory and cognition in line with Landreth's findings.

Results from all four studies were reported as non-peer reviewed technical comments in Science.

\section{Formulation fulmination}

In a published response to the four technical comments, ${ }^{6}$ Landreth's team suggested that differences in drug formulation could explain the discrepancy between the original study and the efforts to replicate it.

For example, Landreth's team noted that the toxicity and lack of efficacy seen by De Strooper's team could be due to the use of a different vehicle. Landreth's team used off-the-shelf Targretin capsules solubilized in water. De Strooper's team used cyclodextrin-based solvents.

Of the four groups, the University of Pittsburgh team used the closest formulation to the one in Landreth's original paper. That team saw the most pronounced results with the drug.

The findings highlight the difficulty in consistently conducting seemingly similar assays in different laboratories. For example, the fact that two of the new teams saw reductions in soluble $A \beta$ and the other two teams did not suggests that subtle methodological differences can lead to highly varied results.

There is little consensus about how changes in biomarkers such as soluble $\mathrm{A} \beta$ or plaque levels in mice relate to human disease. Likewise, it is unclear whether improving mouse learning and memory would lead to meaningful improvements in functional and cognitive endpoints in humans, the clinical standard for AD therapy.

Landreth cofounded ReXceptor Inc. to develop brain-penetrating formulations of bexarotene to treat $\mathrm{AD}$. The company plans to start a Phase Ib trial in healthy volunteers.

"The trial will use the FDA-approved dose of the drug, administered exactly as described on the Eisai label," said Landreth.

The Cleveland Clinic is running a Phase II trial of bexarotene. It has not been disclosed how that formulation differs from ReXceptor's.

Osherovich, L. SciBX 6(21); doi:10.1038/scibx.2013.508

Published online May 30, 2013

\section{REFERENCES}

1. Fitz, N.F. et al. Science; published online May 24, 2013; doi:10.1126/science.1235809

Contact: Radosveta Koldamova, University of Pittsburgh,

Pittsburgh, Pa.

e-mail: radak@pitt.edu

Contact: lliya Lefterov, same affiliation as above

e-mail: iliyal@pitt.edu 


\section{ANALYSIS}

2. Price, A.R. et al. Science; published online May 24, 2013; doi:10.1126/science.1234089

Contact: Kevin M. Felsenstein, University of Florida College of Medicine, Gainesville, Fla. e-mail: kfelsenstein0@ufl.edu

3. Tesseur, I. et al. Science; published online May 24, 2013; doi:10.1126/science.1233937

Contact: Bart De Strooper, Catholic University Leuven, Leuven, Belgium e-mail: bart.destrooper@cme.vib-kuleuven.be

4. Veeraraghavalu, K. et al. Science; published online May 24, 2013; doi:10.1126/science.1235505

Contact: Mathias Jucker, University of Tuebingen, Tuebingen, Germany

e-mail: mathias.jucker@uni-tuebingen.de

Contact: David M. Holtzman, Washington University in St. Louis

School of Medicine, St. Louis, Mo.

e-mail: holtzman@neuro.wustl.edu

Contact: Rudolph E. Tanzi, Harvard Medical School, Cambridge,

Mass.

e-mail: tanzi@helix.mgh.harvard.edu

Contact: Robert Vassar, Northwestern University Feinberg School of Medicine, Chicago, III.

e-mail: r-vassar@northwestern.edu

Contact: Sangram S. Sisodia, The University of Chicago, Chicago, III. e-mail: ssisodia@bsd.uchicago.edu
5. Cramer, P.E. et al. Science 335, 1503-1506 (2012)

6. Landreth, G.E. et al. Science; published online May 24, 2013; doi:10.1126/science. 1234114

Contact: Gary E. Landreth, Case Western Reserve University School of Medicine, Cleveland, Ohio

e-mail: gel2@case.edu

COMPANIES AND INSTITUTIONS MENTIONED

Case Western Reserve University School of Medicine,

Cleveland, Ohio

Catholic University Leuven, Leuven, Belgium

Cleveland Clinic Lou Ruvo Center for Brain Health,

Las Vegas, Nev.

Eisai Co. Ltd. (Tokyo:4523; Osaka:4523),

Tokyo, Japan

Harvard Medical School, Boston, Mass.

Northwestern University Feinberg School of Medicine,

Chicago, III.

ReXceptor Inc., Cleveland, Ohio

The University of Chicago, Chicago, III.

University of Florida College of Medicine,

Gainesville, Fla.

University of Pittsburgh, Pittsburgh, $\mathrm{Pa}$.

University of Tuebingen, Tuebingen, Germany

Washington University in St. Louis School of Medicine, St. Louis, Mo. 